\section{Early European Mathematics}

T he period from 500 to 1000 in Europe is known as the Dark Ages. The legacy of the ancient world was almost forgotten, schooling became infrequent, and the general level of culture remained low. Mathematical activity was generally sparse, but included some writings on the calendar and on finger reckoning.

Revival of interest in mathematics began with Gerbert of Aurillac (938-1003), who trained in Catalonia and may have been the first to introduce the Hindu-Arabic numerals to Christian Europe, using an abacus that he had designed for the purpose. An important figure in the Church, he was crowned Pope Sylvester II in 999.
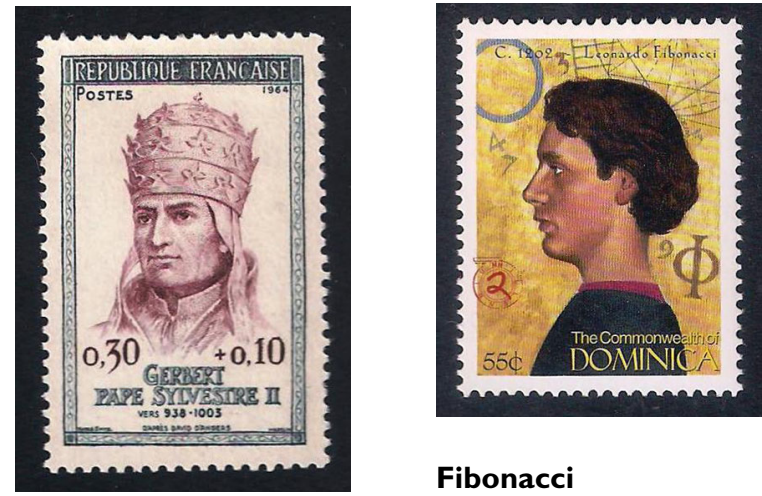

Fibonacci

\section{Gerbert of Aurillac}

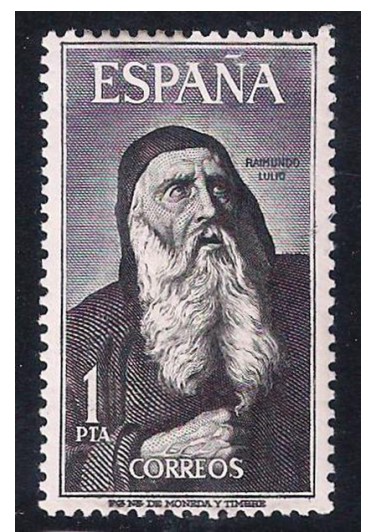

Ramon Lull
Hindu-Arabic methods of calculation were also used by Fibonacci (Leonardo of Pisa) in his Liber Abaci [Book of calculation] of 1202. This celebrated work contained many problems in arithmetic and algebra, including the problem of the rabbits that leads to the "Fibonacci sequence," 1, 1, 2, 3, 5, $8,13, \ldots$, in which each successive term is the sum of the previous pair.

The Catalan mystic Ramon Lull (c.1232-1316) believed that all knowledge can be obtained as mathematical combinations of a fixed number of "divine attributes" (such as power, wisdom, etc.). During the next few centuries, Lull's combinatorial ideas spread through Europe and influenced later mathematicians such as Mersenne and Leibniz.

Other distinguished figures involved with mathematics around this time were Geoffrey Chaucer (1342-1400), author of the Canterbury Tales, who wrote a treatise on the astrolabe, one of the earliest science books to be written in English.

The German philosopher Nicholas of Cusa (or Cusanus) (1401-1464) also wrote several mathematical tracts, invented concave lens spectacles, and attempted the classical problems of trisecting an angle and squaring the circle.

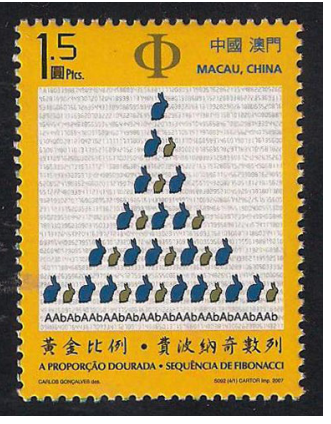

Problem of the rabbits

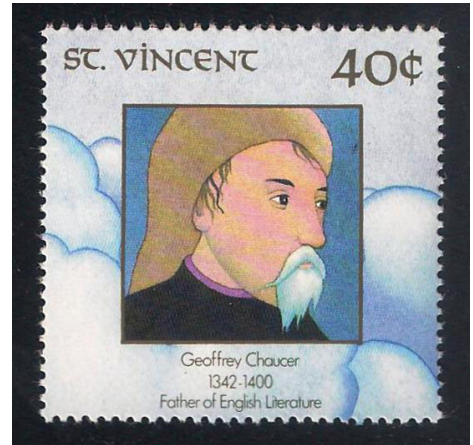

Geoffrey Chaucer

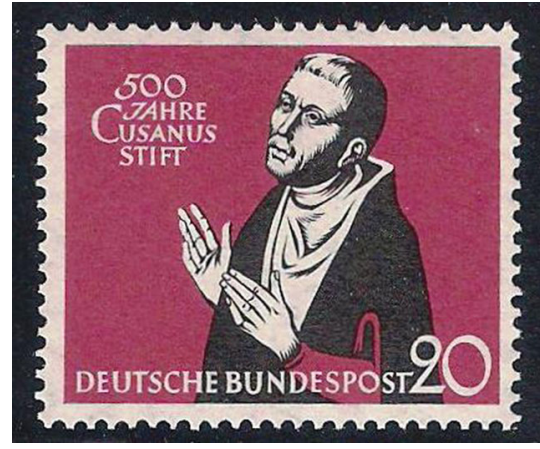

Nicholas of Cusa 